\title{
Knowledge, Awareness, and Eye Care-Seeking Behavior in Diabetic Retinopathy: A Cross-Sectional Study in Jeddah, Kingdom of Saudi Arabia
}

Mashaer O. Fallatah

Received: August 17, 2018 / Published online: September 19, 2018

(C) The Author(s) 2018

\section{ABSTRACT}

Introduction: Diabetes mellitus (DM) is common metabolic disorder that is characterized by increased circulating blood glucose levels. Longterm, continuous hyperglycemia leads to vasculature-related disorders, including those affecting the eyes, such as retinopathy. The objective of this study was to assess the awareness of diabetic complications, specifically diabetic retinopathy, among diabetic patients attending the Jeddah Eye Hospital.

Methods: This was a cross-sectional study targeting the outpatient clinics of Jeddah Eye Hospital for a period of 2 months. A total of 380 participants were randomly selected based on sample size calculations. A closed-ended questionnaire, generated after an extensive literature review, was distributed among the selected individuals. The questions focused on the participants' clinical status of DM, socio-

Enhanced digital features To view enhanced digital features for this article go to https://doi.org/10.6084/ m9.figshare.7039994.

Electronic supplementary material The online version of this article (https://doi.org/10.1007/s40123018-0147-5) contains supplementary material, which is available to authorized users.

M. O. Fallatah $(\bowtie)$

Public Health Sector, Ministry of Health, Jeddah, Saudi Arabia

e-mail: mashaer_F@yahoo.com demographic characteristics, awareness of eye complications secondary to DM, eye screening, and eye care-seeking behavior.

Results: The mean age of the patients was 58.3 (standard deviation 10.9) years, and $52.4 \%$ of patients were female. The majority $(89.7 \%)$ of participants had type II DM (T2DM). The level of awareness was satisfactory $(92.4 \%)$; however, only $10.5 \%$ of participants knew the recommended frequency for eye check-ups. The level of awareness of related complications was directly influenced by education level, source of patient information, place of residence, and frequency of follow-up visits.

Conclusion: The results of this study indicate that although the awareness of diabetes-related eye complications was satisfactory in the patient population, eye care-seeking behavior and frequency of eye check-ups were not optimal. Efforts are needed to promote eye careseeking behavior in this patient group.

Keywords: Awareness; Diabetes; Eye-care-seeking behavior; Retinopathy

\section{INTRODUCTION}

Diabetes mellitus (DM) is the most common metabolic disease worldwide [1]. It is characterized by disturbances in the circulating blood glucose levels that ultimately lead to hyperglycemia. DM is diagnosed based on specific 
circulating blood plasma glucose levels. The most commonly employed diagnostic indicators of DM are fasting plasma glucose levels of $\geq 126 \mathrm{mg} / \mathrm{dl}$, an oral glucose tolerance test result of $\geq 200 \mathrm{mg} / \mathrm{dl}$, checked $2 \mathrm{~h}$ after a glucose loading of $75 \mathrm{~g}$ ( 2 -h plasma glucose), and glycosylated hemoglobin (HbA1c) levels of $\geq$ $6.5 \%[2]$.

According to the World Health Organization, there are currently approximately 280 million diabetic individuals globally, with the expectation that the prevalence of DM will double by the year 2025 [1]. Alarmingly, the areas of the Middle East and North Africa have a higher prevalence of DM than other parts of the world [3], which means that patients in these regions are also at a higher risk of developing diabetesrelated complications. The Kingdom of Saudi Arabia (KSA) is among the top-ten countries with the highest prevalence of DM in the world [4], a dubious honor that is mainly attributed to lifestyle changes [3]. Physical inactivity and a sluggish lifestyle, secondary to economic betterment, are the main contributors to the decreased life expectancy in the KSA [5].

There are two main types of DM, namely, type $1 \mathrm{DM}$ (T1DM) and type $2 \mathrm{DM}$ (T2DM), and these differ in terms of age of onset and pathology. T1DM, or insulin-dependent DM (sometimes known as juvenile diabetes), is a consequence of autoimmune disease. In T1DM, the natural immune system of the body damages beta cells of the pancreas, rendering them unable to produce insulin. In comparison, T2DM, or insulin-independent DM, occurs at a later age and has a multifactorial etiology [6]. T2DM is much more common than T1DM 1 [7].

DM affects the vasculature of the eyes, which leads to various disorders, such as glaucoma, vitreous hemorrhage, cataracts, diabetic retinopathy, and blindness. Diabetic retinopathy, the most common disorder affecting the eyes of patients with DM [8], is a progressive blood vessel disorder of the retina that is becoming one of the main causes of blindness in the age group of 20-60 years worldwide [1]. It is estimated that, worldwide, $15,000-39,000$ patients will lose their vision due to DM [1].

Assessing awareness among diabetics is a necessary educational measure to help in the control of DM. Eye complications due to DM, such as retinopathy, can lead to high social and economic burdens. Currently, information on the level of such awareness in Jeddah City is lacking. Therefore, the aim of this study was to assess the level of awareness of DM complications, specifically eye complications, among adult diabetic patients attending the Jeddah Eye Hospital.

\section{METHODS}

A cross-sectional study was conducted in Jeddah Eye Hospital, which is a government-funded hospital located in Jeddah (the commercial capital of KSA).

The sample size was calculated by using Jeddah Eye Hospital statistics from 2014, which showed that 85,000-95,000 patients visited the outpatient clinics of this hospital during that year. OpenEpi (http://www.openepi.com), an open-source software program, was used to calculate the sample size for a confidence interval (CI) of $95 \%$ and a margin of error of 0.05 , and indicated that 380 participants would be necessary.

Consequently, 380 patients were recruited from the outpatient department of the hospital, either by referral from a primary healthcare center or through an appointment/consultation in the department. Patients were included in the study by choosing the first patient of the day and thereafter every third patient on the appointment list. Visitors of any nationality and gender who attended the outpatient department of Jeddah Eye Hospital during June to July 2015 and who had been previously diagnosed with DM were eligible for inclusion in the study. Patients who were seriously ill, inpatients, and those not able to give valid informed consent were excluded.

A 26-item closed-ended questionnaire was administered to the 380 randomly selected participants (see Electronic Supplementary Material Appendix). The questionnaire was designed after a detailed literature review; the main sources were the Behavioral Risk Factor Surveillance System Questionnaire (2012 and 2014) $[9,10]$ and the questionnaire developed 
by Tajunisah et al. for evaluating awareness of eye complications among patients with T2DM [1]. The questionnaire was translated into Arabic for ease of use by the participants. There were two sets of questions; one set was used to obtain demographic data, the type of DM, modality of treatment, and time between diagnoses and referral to the eye clinic; the second set was used to assess the knowledge of the patient regarding diabetic eye complications, eye screening, and eye care seeking behavior.

The data provided by the completed questionnaires were transferred by the author into an electronic format using the electronic form functionality in Adobe Acrobat X Pro (Adobe Systems Incorporated, Austin, TX, USA) and subsequently exported as an XML file that was analyzed using SAS version 9.4 software (SAS Institute, Cary, NC, USA). The Chi-square test was utilized to test the association of dependent and independent variables and association of all independent variables. $t$ tests were utilized to test the association between continuous variables. $P$ values of $<0.05$ were considered to indicate statistical significance.

\section{Compliance with Ethics Guidelines}

All procedures performed in studies involving human participants were in accordance with the institutional review board (IRB) of Emory University (Atlanta, GA, USA) and with the 1964 Helsinki declaration and its later amendments, or comparable ethical standards. It was classified as public health practice and therefore not subject to review as "human subjects research" (IRB00081503). It was also approved by the local IRB of the Ministry of Health, KSA, (MOH trial registration no. 00611, approval no. A00277). Informed consent was obtained from all individual participants included in the study.

\section{RESULTS}

The mean age of the patients was 58.3 years (standard deviation 10.9 years). Of the 380 participants, 291 resided in Jeddah, and 52.4\% were female (Table 1). Among the total patient group, $89.7 \%$ had T2DM; the remainder had
Table 1 Demographic characteristics of the 380 study participants

\begin{tabular}{|c|c|}
\hline Variables & Value \\
\hline \multicolumn{2}{|l|}{ Age (years) } \\
\hline $0-45$ & $31(8.2)$ \\
\hline $45-55$ & $86(22.6)$ \\
\hline $55-65$ & $154(40.5)$ \\
\hline$\geq 65$ & $109(28.7)$ \\
\hline \multicolumn{2}{|l|}{ Sex } \\
\hline Male & $181(47.6)$ \\
\hline Female & $199(52.4)$ \\
\hline \multicolumn{2}{|l|}{ Nationality } \\
\hline Saudi & $332(87.4)$ \\
\hline Non-Saudi & $48(12.6)$ \\
\hline \multicolumn{2}{|l|}{ Residency } \\
\hline Inside Jeddah & $291(76.6)$ \\
\hline Outside Jeddah & $89(23.4)$ \\
\hline \multicolumn{2}{|l|}{ Primary healthcare center } \\
\hline Followed up & $255(67.1)$ \\
\hline Not followed up & $125(32.9)$ \\
\hline \multicolumn{2}{|l|}{ Educational level } \\
\hline Illiterate & $168(44.2)$ \\
\hline Primary school & $70(18.4)$ \\
\hline Preparatory school & $146(12.1)$ \\
\hline Secondary school & $49(12.9)$ \\
\hline University and higher degree & $47(12.4)$ \\
\hline
\end{tabular}

Values in table are presented as a number with the percentage of total patient population $(N=380)$ in parenthesis

T1DM. In terms of treatment, $41.9 \%$ of participants were on insulin. In total, $91.8 \%$ of patients had visited the doctor for an assessment of their DM before the year in which this study was conducted (Table 2).

In terms of knowledge of DM complications, $92.4 \%$ of the participants were aware that DM 
Table 2 Association between different clinical variables and sex

\begin{tabular}{|c|c|c|c|c|}
\hline Clinical variables & Female & Male & Total & $p$ value \\
\hline \multicolumn{5}{|l|}{ Diabetes type } \\
\hline Type 1 & $15(8.3 \%)$ & $8(4.0 \%)$ & $23(6.1 \%)$ & \multirow[t]{3}{*}{0.217} \\
\hline Type 2 & $159(87.8 \%)$ & $182(91.5 \%)$ & $341(89.7 \%)$ & \\
\hline Unknown & $7(3.9 \%)$ & $9(4.5 \%)$ & $16(4.2 \%)$ & \\
\hline \multicolumn{5}{|l|}{ Use of insulin } \\
\hline Yes & $79(43.9 \%)$ & $79(40.1 \%)$ & $158(41.9 \%)$ & \multirow[t]{2}{*}{0.45} \\
\hline No & $101(56.1 \%)$ & $118(59.9 \%)$ & $219(58.1 \%)$ & \\
\hline \multicolumn{5}{|l|}{ Seen by doctor in past 12 months } \\
\hline Yes & $168(92.8 \%)$ & $181(91.0 \%)$ & $349(91.8 \%)$ & \multirow[t]{2}{*}{0.326} \\
\hline No & $13(7.2 \%)$ & $18(9.0 \%)$ & $31(8.2 \%)$ & \\
\hline \multicolumn{5}{|l|}{ Follow-up recommendation } \\
\hline$<4$ visits in the last 12 months & $76(43.7 \%)$ & $62(31.8 \%)$ & $138(37.4 \%)$ & \multirow[t]{2}{*}{0.015} \\
\hline$\geq 4$ visits in the last 12 months & $98(56.3 \%)$ & $133(68.2 \%)$ & $231(62.6 \%)$ & \\
\hline Glucose check-up frequencies & $31(17.3 \%)$ & $26(13.1 \%)$ & $57(15.1 \%)$ & \multirow[t]{6}{*}{0.196} \\
\hline Never & $46(25.7 \%)$ & $78(39.2 \%)$ & $124(32.8 \%)$ & \\
\hline$<4$ times a day & $6(3.4 \%)$ & $2(1.0 \%)$ & $8(2.1 \%)$ & \\
\hline$>4$ times a day & $31(17.3 \%)$ & $34(17.1 \%)$ & $65(17.2 \%)$ & \\
\hline$<4$ times a month & $61(34.1 \%)$ & $58(29.1 \%)$ & $119(31.5 \%)$ & \\
\hline$>4$ times a month & $4(2.2 \%)$ & $1(0.5 \%)$ & $5(1.3 \%)$ & \\
\hline \multicolumn{5}{|l|}{ Eye screening referral } \\
\hline Yes & $121(33.2 \%)$ & $134(67.3 \%)$ & $255(67.1 \%)$ & \multirow[t]{2}{*}{0.784} \\
\hline No & $60(66.8 \%)$ & $65(32.7 \%)$ & $125(32.9 \%)$ & \\
\hline \multicolumn{5}{|c|}{ Time between diagnosis and eye screening } \\
\hline Between 0 and 10 years & $79(44.6 \%)$ & $73(37.6 \%)$ & $152(41.0 \%)$ & \multirow[t]{3}{*}{0.219} \\
\hline Between 10 and 20 years & $51(28.8 \%)$ & $72(37.1 \%)$ & $123(33.1 \%)$ & \\
\hline$>20$ years & $47(26.6 \%)$ & $49(25.3 \%)$ & $96(25.9 \%)$ & \\
\hline \multicolumn{5}{|l|}{ Wear eyeglasses or contact lenses } \\
\hline Yes & $109(60.2 \%)$ & $129(65.5 \%)$ & $238(62.9 \%)$ & \multirow[t]{2}{*}{0.259} \\
\hline No & $72(39.8 \%)$ & $68(34.5 \%)$ & $140(37.1 \%)$ & \\
\hline
\end{tabular}


Table 2 continued

\begin{tabular}{|c|c|c|c|c|}
\hline Clinical variables & Female & Male & Total & $p$ value \\
\hline \multicolumn{5}{|l|}{ Last eye examination } \\
\hline Never & $14(7.8 \%)$ & $15(7.5 \%)$ & $29(7.6 \%)$ & 0.29 \\
\hline Don't know/not sure & $8(4.4 \%)$ & $4(2.0 \%)$ & $12(3.2 \%)$ & \\
\hline Within past year & $142(78.9 \%)$ & $169(85.0 \%)$ & $311(82.1 \%)$ & \\
\hline$>1$ year & $16(8.9 \%)$ & $11(5.5 \%)$ & $27(7.1 \%)$ & \\
\hline \multicolumn{5}{|l|}{ Retinopathy } \\
\hline Yes & $119(66.1 \%)$ & $134(67.7 \%)$ & $253(66.9 \%)$ & 0.739 \\
\hline No & $55(30.6 \%)$ & $56(28.3 \%)$ & $111(29.4 \%)$ & \\
\hline Don't know/not sure & $6(3.3 \%)$ & $8(4.0 \%)$ & $14(3.7 \%)$ & \\
\hline \multicolumn{5}{|c|}{ HbA1C (glycosylated hemoglobin A1c) } \\
\hline$>6.5 \%$ & $52(96.3 \%)$ & $76(96.2 \%)$ & $128(96.2 \%)$ & 0.978 \\
\hline$<6.5 \%$ & $2(3.7 \%)$ & $3(3.8 \%)$ & $5(3.8 \%)$ & \\
\hline
\end{tabular}

Values in table are presented as a number with the percentage of total patient population $(N=380)$ in parenthesis

can lead to eye disease; $5 \%$ thought that DM has no effect on eyes, whereas $2.6 \%$ did not know anything about diabetes-related eye diseases (Table 3).
Of the participants, $81.7 \%$ agreed to have their eyes checked, even when their blood glucose was in the normal range (Table 4). Similarly, when asked about the frequency of eye check-up visits, $69.5 \%$ of participants answered

Table 3 Knowledge of diabetes-related complications among participants

\begin{tabular}{llll}
\hline Complication & $\begin{array}{l}\text { Yes, patient was aware that } \\
\text { diabetes can lead to eye disease }\end{array}$ & $\begin{array}{l}\text { No, patient was not aware that } \\
\text { diabetes has an effect on eyes }\end{array}$ & $\begin{array}{l}\text { Patient knew nothing about } \\
\text { diabetes-related eye diseases }\end{array}$ \\
\hline $\begin{array}{l}\text { Coronary } \\
\text { artery } \\
\text { disease }\end{array}$ & $246(64.7)$ & $65(17.1)$ & $69(18.2)$ \\
$\begin{array}{l}\text { Stroke } \\
\begin{array}{l}\text { Peripheral } \\
\text { vascular }\end{array}\end{array}$ & $228(60.2)$ & $72(19.0)$ & \\
disease & $61(16.1)$ & $79(20.8)$ \\
$\begin{array}{l}\text { Neuropathy } \\
\text { Eye disease }\end{array}$ & $259(68.5)$ & & $82(21.6)$ \\
Nephropathy & $269(70.8)$ & $67(17.7)$ & $52(13.8)$ \\
\hline
\end{tabular}

Values in table are presented as a number with the percentage of total patient population $(N=380)$ in parenthesis 
Table 4 Knowledge of eye check-up frequency when blood sugar levels are controlled

\begin{tabular}{lc}
\hline $\begin{array}{l}\text { Eye check-up even though blood sugar } \\
\text { levels are normal }\end{array}$ & Values \\
\hline Yes & $308(81.7)$ \\
No & $52(13.8)$ \\
Sometimes & $17(4.5)$ \\
\hline
\end{tabular}

Values in table are presented as a number with the percentage of total patient population $(N=380)$ in parenthesis

that they considered that eye check-ups should be conducted at 6-month intervals, $19.2 \%$ answered that eye check-ups should be performed when there is a problem with vision, while $10.5 \%$ considered that yearly eye checkups were adequate (Table 5).

When asked about the source of information from which they obtained knowledge about the complications of DM, $67.2 \%$ participants answered that health-care workers were their source of information, $18.0 \%$ answered that routes of mass communication provided them with information, while $14.8 \%$ obtained their information from their relatives or friends (Table 6).

The level of awareness about complications of DM was significantly associated with level of education $(p<0.05)$ (Fig. 1$)$. The source of the patient's information was also significantly associated with awareness $(p<0.05)$. Participants who gained their knowledge from mass media had a higher level of awareness than

Table 5 Knowledge about eye check-up frequency

\begin{tabular}{lc}
\hline Eye check-up frequency & Value \\
\hline Every 6 months & $264(69.5)$ \\
Only when vision is affected & $73(19.2)$ \\
Two-yearly interval & $3(0.8)$ \\
Yearly & $40(10.5)$ \\
\hline
\end{tabular}

Values in table are presented as a number with the percentage of total patient population $(N=380)$ in parenthesis
Table 6 Source of knowledge about complications

\begin{tabular}{ll}
\hline Source of information & Value \\
\hline Doctor/nurse/ophthalmologist/optometrist & $254(67)$ \\
Family member/friends/relative with diabetes & $56(14.8)$ \\
Television/radio/newspaper/Internet & $68(18)$ \\
\hline
\end{tabular}

Values in table are presented as a number with the percentage of total patient population $(N=380)$ in parenthesis

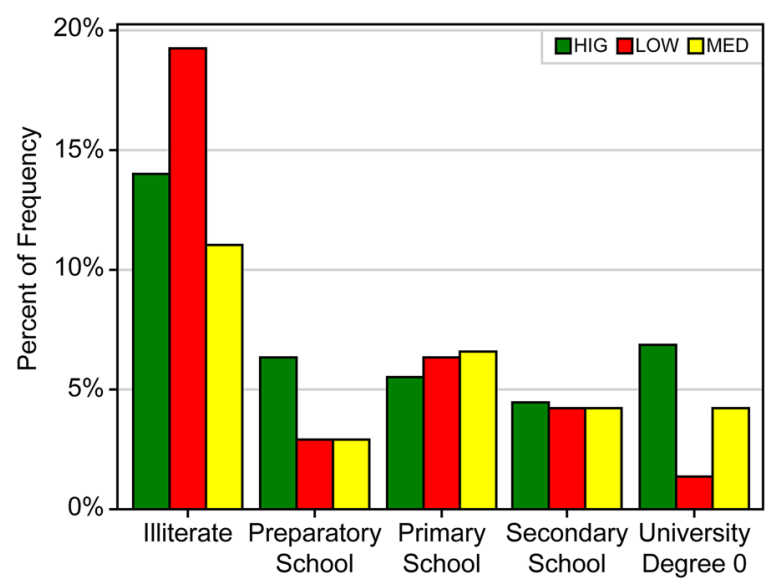

Fig. 1 Level of awareness about complications of diabetes mellitus according to level of education. HIG High level of awareness, $L O W$ low level of awareness, MED medium level of awareness

those who gained their knowledge from healthcare professionals (Fig. 2). The area of residency was also significantly associated with the patient's awareness $(p<0.05)$. Residents of Jeddah had a higher awareness of DM eye complications than those from outside this city.

\section{DISCUSSION}

A study published in 2016 showed that the prevalence of pre-diabetes among the adult population of Jeddah was $9.0 \%$ (8.6\% in women and $9.4 \%$ in men) [11]. The overall prevalence of diabetic retinopathy in the KSA ranges between 33 and $36 \%$ in different regions [12].

The proportion of participants (66.9\%) diagnosed with diabetic retinopathy in this 


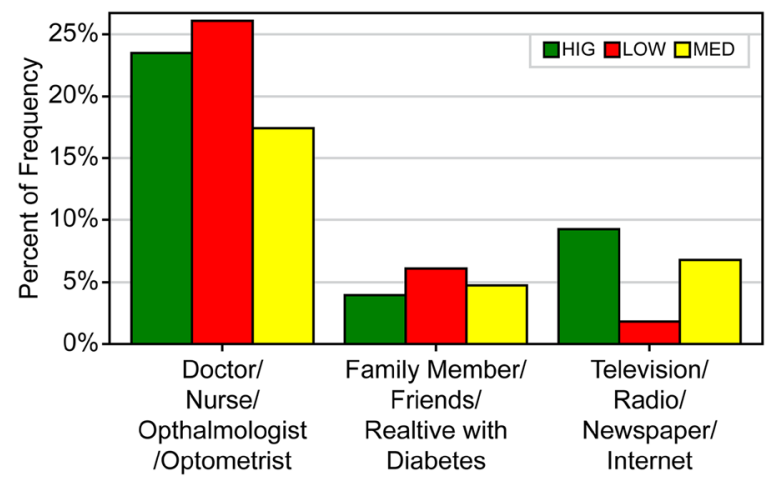

Fig. 2 Level of awareness about complications of diabetes mellitus according to source of knowledge. $H I G$ High level of awareness, $L O W$ low level of awareness, $M E D$ medium level of awareness

study was higher than that reported in 2012 in a study conducted by El-Bab et al. in the city of Al-Madinah Al-Munawarah (36.1\%) [13]. However, $92 \%$ of the participants in the present study were aware of the effects of DM on eyes, which is higher than ever previously reported in a study conducted in the KSA. Tajunishah et al. reported that $86.1 \%$ participants were aware of DM causing eye diseases [1], whereas the authors of a hospital-based study conducted in India concluded that only $51 \%$ people had knowledge of the association between DM and eye diseases [14].

In the present study, follow-up with healthcare professionals (based on recommended guidelines) and level of awareness were significantly associated. In contrast, Addoor and Bhandary reported that among the diabetics attending peripheral diabetic clinics in Melaka, Malaysia, there was no statistically significant effect of previous follow-up visits for DM on the level of awareness [15].

Educated individuals participating in this study had a better understanding of the need for follow-up and the frequency of eye check-ups. Education enhances awareness and thus plays a key role in the development of a healthy society [1]. The patients with secondary or graduate level education in our study were well aware of diabetes-related conditions. Malaysian and South African studies have also demonstrated similar results $[9,16]$. Clearly, individuals who are well educated have better access to different modes of mass communication and are better empowered to understand their health condition.

In the present study, $67.2 \%$ of the patients had obtained information about diabetes-related eye complications from healthcare providers, while $18 \%$ of the patients had obtained this information from mass media. In a U.S. study on Hispanics, around $60 \%$ of the patients had obtained their diabetes-related knowledge from healthcare providers [17]. In the present study it was also evident that although the proportion of individuals obtaining this knowledge from mass media was smaller than that obtaining information from healthcare providers, the former had a better understanding of their disease. This result clearly questions the level of awareness instilled by healthcare providers as it would appear that mass media was more effective in conveying its message across the population. This finding also suggests that healthcare workers should be trained in developing effective communication skills, as they have to deal with many illiterate patients; the percentage of illiterate individuals in this study was $44.2 \%$.

Inadequate awareness of diabetic eye complications is the main barrier preventing patients from having earlier eye check-ups, as indicated by the prevalance of inadequate awareness in the present study (62.8\%). Lack of knowledge was also the main barrier to attending regular check-ups in the above-mentioned Malaysian study [9]. Clearly, an increase in public awareness will lead to a decreased incidence of diabetic retinopathies [5].

\section{Limitations}

Actually the study was conducted from June 1 to July 30, which included the Muslims' month of fasting (Ramadan). Data collection may have been affected by factors related to this observance, such as type of patients showing up for appointments during this month and the reduced working hours. Moreover, the study was a hospital-based study conducted in one specialized eye hospital; a similar study might need to be conducted in a public or non-specialized hospital to validate the results. Very 
limited access to medical records was a barrier to obtaining data on HbA1c levels, and the study relied on participants to report this data, which may have introduced a recall bias.

\section{CONCLUSIONS}

The results of this study show that although a high percentage of the participants were aware of diabetes-related complications in general and eye diseases specifically, they did not have sufficient knowledge to prevent and manage these. The awareness that is instilled via healthcare providers, such as doctors and nurses, is inefficient. Different courses and lectures should be designed for healthcare workers to improve their communication skills. The results reported here indicate that mass media is more effective in instilling awareness and understanding of diabetes-related complications due to its ability to raise important health issues that reflect public concerns. Health messages delivered through mass media should be simple, creative, use different formats, and be repeated many times. It should be noted that these messages are unlikely to lead to the development of skills or to change behaviors, but they can provide the right information, which is a step toward increasing the patient's level of awareness.

\section{ACKNOWLEDGEMENTS}

I would like to acknowledge my parents for their constant motivation and support. I would like to thank my advisor and everyone who has helped and supported me in this research at Emory University. I would also like to thank the participants of the study.

Funding. No funding or sponsorship was received for this study or for the publication of this article. The article processing charges were funded by the author.

Authorship. The named author meets the International Committee of Medical Journal Editors (ICMJE) criteria for authorship for this article, takes responsibility for the integrity of the work as a whole, and has given his approval for this version to be published.

Disclosures. Mashaer O. Fallatah has nothing to disclose.

Compliance with Ethics Guidelines. All procedures performed in studies involving human participants were in accordance with Emory University IRB and with the 1964 Helsinki declaration and its later amendments or comparable ethical standards. It was classified as public health practice and therefore not subject to review as "human subjects research" (IRB00081503), it was also approved by the local IRB of the Ministry of Health, KSA, (MOH trial registration number 00611, approval number A00277). Informed consent was obtained from all individual participants included in the study.

Data Availability. The datasets analyzed during the current study are available from the corresponding author on reasonable request.

Open Access. This article is distributed under the terms of the Creative Commons Attribution-NonCommercial 4.0 International License (http://creativecommons.org/licenses/ by-nc/4.0/), which permits any noncommercial use, distribution, and reproduction in any medium, provided you give appropriate credit to the original author(s) and the source, provide a link to the Creative Commons license, and indicate if changes were made.

\section{REFERENCES}

1. Tajunisah I, Wong PS, Tan LT, Rokiah P, Reddy SC. Awareness of eye complications and prevalence of retinopathy in the first visit to eye clinic among type 2 diabetic patients. Int $\mathrm{J}$ Ophthalmol. 2011;4(5):519-24.

2. American Diabetes Association. Standards of medical care in diabetes. Diabetes Care. 2016;39[Suppl 1]:1-142.

3. Sherif S, Sumpio BE. Economics development and diabetes prevalence in MENA countries: Egypt and Saudi Arabia comparison. World J Diabetes. 2015;6(2):304-11. 
4. Aldebasi Y, Reddy P, Nair V, Ahmed M. Screening for diabetic retinopathy: the optometrist's perspective. Clin Optometry. 2015;7:1-14.

5. Kadri R. Awareness of diabetic and hypertensive eye disease in public. Int $\mathrm{J}$ Biol Med Res. 2013;2(2):533-5.

6. Alslail FY. Cardiovascular risk profiles of adults with type 2 diabetes treated at urban hospitals in Riyadh, Saudi Arabia. J Epidemiol Glob Health. 2016;6:29-36.

7. Cockram CS. The epidemiology of diabetes mellitus in Asia Pacific region. Hong Kong Med J. 2000;6(1):43-52.

8. Bodunde OT, Odusan O. Awareness of ocular complications of diabetes among diabetic patients in a tertiary hospital in western Nigeria. J Dent Med Sci. 2014;13(6):9-12.

9. Centers for Disease Control and Prevention (CDC). Behavioral risk factor surveillance system survey data. U.S. Department of Health and Human Services, Centers for Disease Control and Prevention, Atlanta, Georgia; 2012. http://www.cdc.gov/brfss.

10. Centers for Disease Control and Prevention (CDC). Behavioral risk factor surveillance system survey data. U.S. Department of Health and Human Services, Centers for Disease Control and Prevention, Atlanta, Georgia; 2014. http://www.cdc.gov/brfss.

11. Bahijri SM, Jambi HA, Al Raddadi RM, Ferns G, Tuomilehto J. The prevalence of diabetes and prediabetes in the adult population of Jeddah, Saudi Arabia-a community-based survey. PLoS One. 2016;11(4):e0152559.

12. Hajar S, Hazmi A, Wasli M. Prevalence and causes of diabetic retinopathy in Southern Saudi Arabia. Saudi Med J. 2015;36(40):449-55.

13. El-Bab M, Shawky N, Al-Sisi A, Akhtar M. Retinopathy and risk factors in diabetic patients from Al-Madinah Al-Munawarah in the Kingdom of Saudi Arabia. Clin Ophthalmol. 2012;6:269-76.

14. Rani PK, Raman R (2008) Knowledge of diabetes and diabetic retinopathy among rural populations in India, and the influence of knowledge of diabetic retinopathy on attitude and practice. Rural Remote Health 2008;8(3):838. http://www.rrh.org.au.

15. Addoor KR, Bhandary SV. Assessment of awareness of diabetic retinopathy among the diabetics attending the peripheral diabetic clinics in Melaka, Malaysia. Med J Malaysia. 2011;66(1):48-52.

16. Cockburn N, Joubert F. Awareness of diabetic retinopathy among diabetics in the Cape Town Metropole. MMed thesis. Cape Town: University of Cape Town. 2014. https://open.uct.ac.za/bitstream/ item/6586/thesis_hsf_2014_joubert_f.pdf?sequence $=1$.

17. Munoz B, Rosario E. Knowledge of diabetic eye disease and vision care guidelines among Hispanic individuals in Baltimore with and without diabetes. Arch Ophthalmol. 2008;126(7):968-74. 